\title{
Electron capture on iron group nuclei
}

\author{
D.J. Dean ${ }^{1,2}$, K. Langanke ${ }^{3}$, L. Chatterjee ${ }^{1,2,4}$, P.B. Radha ${ }^{5}$, and M. R. Strayer ${ }^{1}$ \\ ${ }^{1}$ Physics Division, Oak Ridge National Laboratory, P.O. Box 2008 \\ Oak Ridge, Tennessee 37831-6373 USA \\ ${ }^{2}$ Department of Physics and Astronomy, University of Tennessee, Knoxville, Tennessee, 37996 \\ ${ }^{3}$ Institute for Physics and Astronomy, University of Aarhus, Denmark \\ and Theoretical Center for Astrophysics, University of Aarhus, Denmark \\ ${ }^{4}$ Cumberland University, Lebanon, Tennessee 37087 \\ ${ }^{5}$ Laboratory for Laser Energetics, University of Rochester, 250 E. River Road, \\ Rochester, NY 14623
}

(November 13, 2017)

\begin{abstract}
We present Gamow-Teller strength distributions from shell model Monte Carlo studies of $f p$-shell nuclei that may play an important role in the precollapse evolution of supernovae. We then use these strength distributions to calculate the electron-capture cross sections and rates in the zero-momentum transfer limit. We also discuss the thermal behavior of the cross sections. We find large differences in these cross sections and rates when compared to the naive single-particle estimates. These differences need to be taken into account for improved modeling of the early stages of type II supernova evolution.
\end{abstract}

PACS numbers: 26.50.+x, 23.40.-s, 21.60Cs, 21.60Ka 


\section{INTRODUCTION}

The impact of nuclear structure on astrophysics has become increasingly important, particularly in the fascinating and presently unsolved problem of type-II supernovae explosions. The possibility to detect neutrinos ejected before the infalling matter reaches the neutrino trapping density [1] will allow us, for the first time, to understand whether models of the precollapse evolution are in reasonable agreement with observation. These studies will also shed light on the effective electron-to-proton ratio in the early stages of the collapse.

Key inputs for the precollapse scenario are the electron capture cross sections and rates for iron group nuclei [2,3]. The core of a massive star at the end of hydrostatic burning is stabilized by electron degeneracy pressure as long as its mass does not exceed the appropriate Chandrasekhar mass $M_{C H}$. If the core mass exceeds $M_{C H}$, electrons are captured by nuclei [2]. Thus, the depletion of the electron population due to capture by nuclei is a crucial factor determining the initial collapse phase.

The reduction of the electroweak interaction matrix element to the zero momentum transfer limit for the nuclear sector leads directly to the Gamow-Teller operator as a primary ingredient in electron-capture cross section calculations. The Gamow-Teller (GT) properties of nuclei in the iron region of the periodic table are known to be crucial for supernova physics [4]. For many of the nuclei that are present in this early stage of the presupernova [3], GT transitions contribute significantly to the electron capture cross sections. Due to insufficient experimental information, the $\mathrm{GT}_{+}$transitions, which change protons into neutrons, have so far been treated only qualitatively in presupernova collapse simulations, assuming the $\mathrm{GT}_{+}$ strength to reside in a single resonance whose energy relative to the daughter ground state has been parametrized phenomenologically [4]; the total $\mathrm{GT}_{+}$strength has been taken from the single particle model. Recent $(n, p)$ experiments [5], however, show that the $\mathrm{GT}_{+}$ strength is fragmented over many states, while the total strength is significantly quenched compared to the single particle model. A recent update of the $\mathrm{GT}_{+}$rates for use in supernova simulations assumed a constant quenching factor of 2 [3], and included the known low-lying transitions in the calculations of the rates.

In the presupernova collapse, electron capture on $p f$-shell nuclei proceeds at temperatures between $300 \mathrm{keV}$ and $800 \mathrm{keV} \mathrm{[3].} \mathrm{While} \mathrm{presupernova} \mathrm{collapse} \mathrm{studies} \mathrm{took} \mathrm{into}$ account the temperature effects on the electron capture rate induced by changes in the electron distribution, potential effects on the Gamow-Teller strength distribution have been neglected, and use was made of the extreme shifting assumption [10] which states that the centroid corresponding to each parent excited state is shifted upward in the daughter nucleus by the excitation energy of the parent state. As experiments measure only the ground state distribution, this hypothesis has necessarily to be tested theoretically, and it has been found approximately valid in a restricted shell model study [11,12]; however, the recent SMMC calculations of Ref. [13] clearly indicate changes of the Gamow-Teller strength distribution with temperature. Thus it appears desirable to take possible changes of the Gamow-Teller strength distribution with temperature also into account when calculating presupernova electron capture rates. We will consistently do this in this paper.

In contrast to previous studies, the calculations presented in this paper solve the full shell model problem for the Gamow-Teller strength distributions in the $0 \hbar \omega f p$-shell space using a realistic residual interaction. We use the shell model Monte Carlo (SMMC) approach to 
find the Gamow-Teller strength distributions. SMMC has the added advantage that it treats nuclear temperature exactly. These distributions are then used to calculate the electroncapture cross section as a function of the incident energy of the electron. We also calculate the electron-capture rates assuming a relativistic electron gas and a variety of electron chemical potentials. In Section II we discuss the methods used in these calculations, while in Section III we present our results. A discussion and conclusion follow in Section IV.

\section{THEORETICAL BACKGROUND}

\section{A. Shell Model Monte Carlo techniques}

The SMMC method is based on a statistical formulation of the nuclear many-body problem. In the finite-temperature version of this approach, an observable is calculated as the canonical expectation value of a corresponding operator $\hat{\mathcal{A}}$ at a given nuclear temperature $T_{N}$, and is given by [14] 17

$$
\langle\hat{\mathcal{A}}\rangle=\frac{\operatorname{Tr}_{\mathrm{A}}[\hat{\mathcal{A}} \hat{U}]}{\operatorname{Tr}_{\mathrm{A}}[\hat{U}]}
$$

where $\hat{U}=\exp (-\beta \hat{H})$ is the imaginary-time many-body propagator, $\operatorname{Tr}_{\mathrm{A}} \hat{U}$ is the canonical partition function for $A$ nucleons, $\hat{H}$ is the shell model Hamiltonian, and $\beta=1 / T_{N}$ is the inverse temperature.

The SMMC canonical expectation values are based on the discretization of the manybody propagator, $\exp (-\beta \hat{H})$, into a finite number of imaginary time slices, $N_{t}$, each of duration $\Delta \beta=\beta / N_{t}$. At each time slice the many-body propagator is linearized via the Hubbard-Stratonovich transformation [18,19]; observables are thus expressed as path integrals of one-body propagators in fluctuating auxiliary fields. The integration is carried out by a Metropolis random walk [20]. To circumvent the sign problem encountered in the SMMC calculations with realistic interactions, we use the extrapolation procedure outlined in Refs. 21,22.

Yet another, but distinct, source of the sign problem is an odd number of nucleons in the canonical expectation values [17]. We overcome this problem by a number-projection technique, first employed in [23] and subsequently used in [17], that allows us to extract information concerning odd- $A$ nuclei from the neighboring even-even system. The partition function for the odd- $A$ nucleus $A^{\prime}$ is given by

$$
Z_{A^{\prime}}=\int \mathcal{D}[\sigma] G(\sigma) \operatorname{Tr}_{A^{\prime}} U_{\sigma}=\int \mathcal{D}[\sigma] G(\sigma) \operatorname{Tr}_{A} U_{\sigma} \frac{\operatorname{Tr}_{A^{\prime}} U_{\sigma}}{\operatorname{Tr}_{A} U_{\sigma}}
$$

where we have introduced the trace over the even-even nucleus $A$. The integration is over all auxiliary field variables, $\sigma$, and $G(\sigma)$ is a Gaussian factor that arises when the HubbardStratonovitch transformation is employed. We define our Metropolis sampling weight as $W(\sigma)=G(\sigma) \operatorname{Tr}_{A} U_{\sigma}$, and in the case of number projection, the Monte Carlo sign becomes $\Phi(\sigma)=\operatorname{Tr}_{A^{\prime}} U_{\sigma} / \operatorname{Tr}_{A} U_{\sigma}$, which is small, but fairly stable against fluctuations at least down to $T_{N}=0.8 \mathrm{MeV}$ for realistic interactions. We project from the nearest even-even system with $A^{\prime}+1$ particles. 
The SMMC method is also used to calculate the response function $R_{\mathcal{A}}(\tau)$ of an operator $\mathcal{A}$ at an imaginary-time $\tau$. The response describes the dynamical behavior of the nucleus under the influence of the operator, and contains information about the nuclear spectrum. This is seen by using a spectral distribution of initial and final states $|i\rangle,|f\rangle$ with energies $E_{i}$ and $E_{f}$

$$
R_{\mathcal{A}}(\tau) \equiv\left\langle\hat{\mathcal{A}}^{\dagger}(\tau) \hat{\mathcal{A}}(0)\right\rangle=\frac{\operatorname{Tr}_{A}\left[e^{-(\beta-\tau) \hat{H}} \hat{\mathcal{A}}^{\dagger} e^{-\tau \hat{H}} \hat{\mathcal{A}}\right]}{\operatorname{Tr}_{A}\left[e^{-\beta \hat{H}}\right]}=\frac{\sum_{i f}\left(2 J_{i}+1\right) e^{-\beta E_{i}} e^{-\tau\left(E_{f}-E_{i}\right)}|\langle f|\hat{\mathcal{A}}| i\rangle|^{2}}{\sum_{i}\left(2 J_{i}+1\right) e^{-\beta E_{i}}}
$$

Note that the total strength for the operator is given by $R(\tau=0)$. The strength distribution

$$
S_{\mathcal{A}}(E)=\frac{\sum_{i f} \delta\left(E-E_{f}+E_{i}\right)\left(2 J_{i}+1\right) e^{-\beta E_{i}}|\langle f|\hat{\mathcal{A}}| i\rangle|^{2}}{\sum_{i}\left(2 J_{i}+1\right) e^{-\beta E_{i}}}
$$

is related to $R_{\mathcal{A}}(\tau)$ by a Laplace Transform,

$$
R_{\mathcal{A}}(\tau)=\int_{-\infty}^{\infty} S_{\mathcal{A}}(E) e^{-\tau E} d E .
$$

Note that here $E$ is the energy transfer within the parent nucleus, and that the strength distribution $S_{G T+}(E)$ has units of $\mathrm{MeV}^{-1}$.

\section{B. Weak interaction cross sections in nuclei}

Electron capture by nuclei is mediated by the electro-weak charged current. At energies appropriate for the pre-collapse supernova, the $\mathrm{W}$-boson propagator may be collapsed to point coupling and the weak vertex represented by the (V-A) coupling with the universal strength $g_{\mathrm{wk}}$. The complexity of computing these channels for electron capture arises from the need to treat both the weak sector and the details of the nuclear structure effects with suitable finesse to obtain reliable cross sections.

Starting from the exact expression for the semi-leptonic process corresponding to electron capture, one can approximate the three-momentum transfer to the nucleus to be zero in the nuclear sector corresponding to computing the nuclear matrix elements between initial and final states. This allows us to calculate and predict the nuclear sector with a high level of accuracy. The lepton traces and kinematics are still included correctly as is energy conservation between the initial and final states. The sacrifice of the magnitude of the momentum transfer to the nucleus in the nuclear sector is reasonably well justified at the energy domain operative in the precollapse stellar core where energies are low and the energy transfer from the incident charged lepton to the nucleus is mainly used for internal nuclear excitation.

Electrons with energy $E_{e}$ interact with the nucleus through the weak interaction. For charge exchange interactions

$$
e^{-}+A(N, Z) \rightarrow A(N+1, Z-1)+\nu_{e^{-}} .
$$


The parent nucleus absorbs a part of the incident energy and the neutrino carries the remaining energy $\varepsilon_{\nu}$. The energy absorbed by the parent is given by the difference between the initial and final nuclear states $E=E_{f}-E_{i}$, and the states are labeled by $\{|f\rangle,|i\rangle\}$, respectively. Energy conservation requires that $E_{e}=E+\varepsilon_{\nu}$. The details of the derivation of the matrix element are given in [24. The angular cross section follows as

$$
\frac{d \sigma}{d \Omega}=\frac{\varepsilon_{\nu}^{2} V}{(2 \pi)^{2}}\left[\sum_{\text {leptonspins }} \frac{1}{2 J_{i}+1} \sum_{M_{f} M_{i}}\left|\left\langle f\left|\hat{H}_{W}\right| i\right\rangle\right|^{2}\right]
$$

where $\hat{H}_{W}$ is the weak interaction Hamiltonian, $J_{i}\left(J_{f}\right)$ is the initial (final) spin of the nuclear state, and $M_{i}, M_{f}$ are projections of the angular momentum of the initial and final states of the nucleus. In the limit of small three-momentum transfer, the partial cross section for electron capture between fixed initial and final states at a given incident electron energy reduces to

$$
\sigma_{f i}\left(E_{e}\right)=\frac{6\left(E_{e}-E\right)^{2} g_{\mathrm{wk}}^{2}}{\pi\left(2 J_{i}+1\right)}\left|\left\langle f\left\|\hat{\mathcal{L}}_{1}\right\| i\right\rangle\right|^{2}
$$

where $g_{\mathrm{wk}}=1.1661 \times 10^{-5} \mathrm{GeV}^{-2}$ is the weak coupling constant, and

$$
\hat{\mathcal{L}}_{1 M}=\frac{i}{\sqrt{12 \pi}} G_{A} \sum_{i=1}^{A} \tau_{+}(i) \sigma_{1 M}(i) .
$$

Here $G_{A}$ is the axial vector form-factor which at zero momentum is $G_{A}=1.25$. The GamowTeller operator is $\mathbf{G} \mathbf{T}_{+}=\sum_{i} \tau_{i}^{+} \sigma_{i}$, where $\tau_{i}^{+}$is the isospin raising operator, and $\sigma_{i}$ is the Pauli spin operator for nucleon $i$. The Clebsch-Gordon coefficients that arise when applying the Wigner-Eckart theorem give selection rules for this operator that require transitions from an initial state with quantum numbers $\left(J_{i}, T_{i}, \Pi_{i}\right)$ to a final state $\left(J_{f}, T_{f}, \Pi_{f}\right)$ such that $\Delta J=J_{f}-J_{i}=0,1$, but no $J_{f}=J_{i}=0, \Delta T=T_{f}-T_{i}=0,1$, and $\Pi_{f}=\Pi_{i}$.

Our previous studies showed that the experimentally observed quenching of the total GT strength is consistently reproduced by the correlations within the full $p f$-shell if a renormalization of the spin operator by the factor 0.8 is invoked [25,26]. The same renormalization factor had already been deduced from sd-shell [27] and $p f$-shell nuclei with $A \leq 49$ 28, 29 and thus appears to be universal. In this work, we also renormalize the spin operator by $0.8, \sigma_{\text {eff }}=0.8 \sigma_{1}$.

In order to obtain the total cross section at a given incident electron energy, one must sum over all initial and final states in Eq. (8). We are generally interested in situations when the initial nucleus is at a finite temperature, so that the initial state sum needs to be weighted by the appropriate statistical factors. Thus, the total cross section is

$$
\begin{aligned}
\sigma\left(E_{e}\right) & =\sum_{i f} \frac{\left(2 J_{i}+1\right) \exp \left(-\beta E_{i}\right)}{Z_{A}} \sigma_{f i}\left(E_{e}\right) \\
& =\frac{6 g_{\mathrm{wk}}^{2}}{\pi} \int d \xi\left(E_{e}-\xi\right)^{2} \sum_{i f} \frac{\exp \left(-\beta E_{i}\right)}{Z_{A}} \frac{G_{A}^{2}}{12 \pi}\left|\left\langle f\left\|\sum_{i=1}^{A} \tau_{+}(i) \sigma_{\mathrm{eff}}(i)\right\| i\right\rangle\right|^{2} \delta\left(\xi-E_{f}+E_{i}\right) .
\end{aligned}
$$

Using the SMMC expression for the Gamow-Teller strength distributions and shifting the energy scale in (4) by an appropriate Coulomb correction and the proton-neutron mass splitting, the total cross section can be recast into the form 


$$
\sigma\left(E_{e}\right)=6 g_{\mathrm{wk}}^{2} \int d \xi\left(E_{e}-\xi\right)^{2} \frac{G_{A}^{2}}{12 \pi} S_{G T_{+}}(\xi) F\left(Z, E_{e}\right) .
$$

Here we have additionally accounted for the distortion of the electron's wave function due to the Coulomb field of the nucleus by introducing the Fermi function $F\left(Z, E_{e}\right)=$ $2 \pi \eta /[\exp (2 \pi \eta)-1]$ where $\eta=Z \alpha / \hat{\beta}, \alpha$ is the fine-structure constant, $\hat{\beta}=|\vec{\beta}|$ is the magnitude of the velocity of the incoming electron, and $Z$ is the charge of the parent nucleus.

The presupernova electron capture rate $\lambda_{\mathrm{ec}}$ is then given by folding the total cross section with the flux of a degenerate relativistic electron gas:

$$
\lambda_{\mathrm{ec}}=\frac{\ln 2}{6163 \mathrm{sec}} \int_{0}^{\infty} d \xi S_{\mathrm{GT}}\left(\xi, T_{N}\right) \frac{c^{3}}{\left(m_{e} c^{2}\right)^{5}} \int_{\mathcal{L}}^{\infty} d p p^{2}\left(-\xi+E_{e}\right)^{2} \frac{F\left(Z, E_{e}\right)}{1+\exp \left[\beta_{e}\left(E_{e}-\mu_{e}\right)\right]},
$$

where $1 / \beta_{e}, p$, and $\mu_{e}$ are the electron temperature, momentum, and chemical potential, and $\mathcal{L}=\left(Q_{i f}^{2}-m_{e}^{2} c^{4}\right)^{1 / 2}$ for $Q_{i f} \leq-m_{e} c^{2}$, and 0 otherwise, and $Q_{i f}=-E=E_{i}-E_{f}$ is the energy difference between the nuclear states $i$ and $f$, respectively. We have indicated that the Gamow-Teller distribution is calculated at the finite nuclear temperature $T_{N}$ which, in principle, is the same as the one of the electron gas $T_{e}=1 / \beta_{e}$. However, we will study below the uncertainties introduced in the electron capture rate due to the temperature dependence of the Gamow-Teller distribution by varying $T_{N}$ for fixed $T_{e}$.

\section{RESULTS}

\section{A. Validation}

The SMMC calculations of Gamow-Teller strength distributions were detailed in Ref. [13]. In the present work, we have calculated several more nuclei in addition to those found in Ref. [13]. Each even-even nucleus is calculated at a temperature of $T_{N}=0.5 \mathrm{MeV}$, while the $g$-extrapolation required to circumvent the SMMC sign problem allows only to cool odd- $A$-nuclei down to a temperature of $T_{N}=0.8 \mathrm{MeV}$. We use a $\Delta \beta=0.03125 \mathrm{MeV}^{-1}$, and approximately four-thousand samples per extrapolation Hamiltonian. We use the KB3 residual interaction [30] which is well suited for full $0 \hbar \omega$ studies throughout the lower $p f$ shell region [26]. Using the numerical techniques described in Ref. [13, 17], we construct a strength distribution from the response function of the Gamow-Teller operator. Recall from Ref. [13] that good agreement was found between experiment and theory for those nuclei that had been experimentally investigated.

In order to demonstrate and validate that our SMMC odd- $A$ projection procedure gives reasonable results for the strength distributions at finite temperatures, we compare our SMMC results in ${ }^{45} \mathrm{Sc}$ to standard shell model diagonalization calculations. ${ }^{45} \mathrm{Sc}$ does not actually play a role during the presupernova collapse. Its choice is, however, dictated as it is the heaviest odd- $A$ nucleus for which state-by-state calculations of the Gamow-Teller strength distribution at finite temperature in the complete $p f$-shell are feasible. Using Eq. 3 above, we calculated $S_{G T_{+}}\left(E, T_{N}\right)$ within the diagonalization approach for all initial states in ${ }^{45} \mathrm{Sc}$ up to an excitation energy of $4.68 \mathrm{MeV}$. At a nuclear temperature of $0.8 \mathrm{MeV}$, the last excited state considered contributes less than $0.09 \%$ to the partition function. Figure 1 compares the thermal distribution (dotted histogram) obtained by diagonalization with 
the SMMC result (solid line) calculated at the same temperature $T_{N}=0.8 \mathrm{MeV}$. The distributions are plotted as function of the nuclear energy transfer $E=E_{f}-E_{i}$ required in (12). (The Coulomb energy of the final state has been corrected using the shift as defined in [25].) One observes that the SMMC calculation reproduces both the position and the width of the thermal distribution very well. The diagonalization calculation gives a total strength of 0.60 , while the SMMC result is $0.52 \pm 0.08$.

To study the temperature dependence of the Gamow-Teller strength distribution we have performed the diagonalization calculations at different temperatures $T_{N}=0 ., 0.3 \mathrm{MeV}$ and $0.8 \mathrm{MeV}$ The results are also given in Fig. 1 . At first we note that the $T=0$ distribution can be compared to the experimental data of Ref. [32]. The total calculated strength $(0.52 \pm 0.08)$ agrees well with the measurement ( 0.48 up to excitation energies of $9 \mathrm{MeV}$; the observed strength at higher excitation energies is likely due to contributions outside the present model space [28]). However, the Gamow-Teller peak of the experimental distribution is about 1 $\mathrm{MeV}$ higher in energy than the calculation, which makes the agreement between data and shell model calculation worse than in the other $p f$-shell nuclei [26,13]. With increasing temperature the Gamow-Teller distributions are broadened and its center is shifted slightly to lower transfer energies. Both effects have already been noticed in the SMMC studies of [13]. The broadening is mainly due to the fact that the number of states contributing to the thermal ensemble increases. The energy shift is caused by the decrease of pairing energy in the higher excited states. We mention that the large peak seen in the distributions at $T_{N}=0$ and $0.3 \mathrm{MeV}$ corresponds principally to ${ }^{45} \mathrm{Sc}(J=7 / 2) \rightarrow{ }^{45} \mathrm{Ca}(J=5 / 2)$ transitions. In the distribution at $T_{N}=0.8 \mathrm{MeV}$ this peak appears lower in energy at $E \approx 5 \mathrm{MeV}$ and its strength is noticeably reduced.

We also want to understand how the electron capture cross section (11), as calculated with the SMMC Gamow-Teller strength distribution at $T_{N}=0.8 \mathrm{MeV}$, compares to the one obtained from the respective diagonalization results. The middle plot in Fig. 1 shows that the SMMC result reproduces the thermal diagonalization data down to $E_{e}=10 \mathrm{MeV}$ very closely, and is within $30 \%$ of the exact result down to $E_{e}=5 \mathrm{MeV}$. At very low electron energies the cross section becomes very sensitive to the discrete level structure of the Gamow-Teller transitions. These weak transitions are not well reproduced by the SMMC calculation leading to a noticeable underestimate of the cross section within the SMMC approach. However, the cross section is already very low at these electron energies and, as we will see next, this shortcoming of the SMMC calculation is not too important for the electron capture rate. Note that the standard shell model calculations yield almost oscillatory behavior at low $E_{e}$ (dashed line), which reflects its discrete level structure at low excitation energies. For completeness, we have also plotted the electron capture cross sections calculated for the Gamow-Teller distributions at $T_{N}=0$ and $T_{N}=0.3 \mathrm{MeV}$. We observe that differences in the distribution only affect the cross section at rather low electron energies.

The quantity of interest for presupernova studies is the electron capture rate (12). In the lower panel of Fig. 1 we compare the rates, calculated from the SMMC and from the diagonalization Gamow-Teller strength distributions at $T_{N}=0.8 \mathrm{MeV}$, as a function of electron chemical potential. The agreement is very satisfying for the entire range $\mu_{e} \leq 10$ $\mathrm{MeV}$ indicating that the differences between the SMMC and diagonalization electron capture cross sections at low electron energies are washed out when folded with the electron flux. 
As mentioned above, SMMC calculations with realistic interactions cannot be performed for odd- $A$ nuclei at temperatures below $T_{N}=0.8 \mathrm{MeV}$. As the relevant temperature during the early stage of the presupernova collapse is lower, we have investigated the inaccuracy introduced in the electron capture rates if the Gamow-Teller distribution at these lower temperatures is approximated by the one at $T_{N}=0.8 \mathrm{MeV}$. Choosing the extreme case, $T_{N}=0.3$, we have performed one consistent calculation of the electron capture rate using the appropriate electron flux and Gamow-Teller strength distribution (determined within the diagonalization approach). This result is compared with a calculation in which the GamowTeller distribution is approximated by the SMMC distribution calculated at $T_{N}=0.8 \mathrm{MeV}$. We observe a maximal difference of a factor 4 for $\mu_{e}<10 \mathrm{MeV}$ which mainly reflects deviations in the Gamow-Teller distribution at low energies. Thus, it can be reduced if the weak discrete transitions which are experimentally known are added by hand to the SMMC results, following the procedure of [4. Obviously the present SMMC approach becomes increasingly more accurate for later stages of the collapse where the temperature is higher and for even-even parent nuclei where SMMC calculations at the relevant temperature are possible.

Finally, we want to study how much the statistical Monte Carlo uncertainties and the errors related to the inversion technique used to determine the Gamow-Teller distribution from the response function (5) affect the electron capture rates. The major uncertainty introduced in our Gamow-Teller strength distribution is in the position of the centroid which has an error of about $\pm 0.5 \mathrm{MeV}$. In Fig. 2 we show how much such a change would affect $\lambda_{\text {ec }}$. Decreasing (increasing) the position of the centroid by $0.5 \mathrm{MeV}$ increases (decreases) the rate by an approximate factor of $1.4(1.3)$.

\section{B. Electron capture results}

Ref. [13 presented SMMC Gamow-Teller distributions for those $p f$-shell nuclei in the iron mass region for which experimental data are available $\left({ }^{54,56} \mathrm{Fe},{ }^{58,60,62,64} \mathrm{Ni},{ }^{51} \mathrm{~V},{ }^{55} \mathrm{Mn}\right.$ and $\left.{ }^{59} \mathrm{Co}\right)$. The agreement between theory and data was very satisfying in all cases, and it was concluded that the SMMC approach has the predictive power to estimate the GamowTeller strength distribution for nuclei for which no data exist. We have extended the study of Ref. [13 by performing SMMC calculations for several of those $p f$-shell nuclei which are of interest for the presupernova collapse: ${ }^{55,57} \mathrm{Co},{ }^{56} \mathrm{Ni},{ }^{50,52} \mathrm{Cr},{ }^{55,58} \mathrm{Fe},{ }^{56} \mathrm{Ni}$, and ${ }^{50} \mathrm{Ti}$. The Gamow-Teller distributions for these nuclei are plotted in Fig. 3 as a function of energy transfer $E=E_{f}-E_{i}$. As for ${ }^{45} \mathrm{Sc}$, the Coulomb correction has been performed following the prescription given in Ref. [25]. Each Gamow-Teller distribution has been interpolated onto a fine energy grid with a spline function. The spline function was then used to calculate the cross section using the formulae described in Section II.

Before presenting results for the electron capture cross sections and rates, we note that our approach, as presented in Section II, implicitly assumes that the calculation reproduces the mass splitting between parent and daughter nucleus correctly. As has been shown in 26,25 the KB3 interaction indeed describes masses of $p f$-shell nuclei reasonably well (typically within $500 \mathrm{keV}$ of the data; as the deviations have a systematical trend the inaccuracy on the relevant mass splitting between parent and daughter should be even smaller). Many of the SMMC masses presented in [25] have very recently also been calculated within the 
conventional diagonalization approach, and the SMMC results (after subtraction of the appropriate internal excitation energy) have been confirmed in all cases [33]. These studies, however, clearly showed [25,33] that the KB3 interaction overbinds the $f_{7 / 2}$ subshell closure. This shortcoming can be circumvented [34 by using the FDP6 interaction [35]. However, this force does not reproduce the Gamow-Teller strength correctly [36]. Weighing the advantages and disadvantages of the two interactions, we have chosen the KB3 interaction with the price that we had to shift the energy scale of the Gamow-Teller distributions in order to correct for the $f_{7 / 2}$ subshell overbinding. As can be seen in Ref. [13], after correction the SMMC calculation reproduces the observed Gamow-Teller distributions in ${ }^{54} \mathrm{Fe}$ and the various $\mathrm{Ni}$ isotopes very well.

In Table 1 we compare the SMMC results for the Gamow-Teller peak in the daughter nucleus with the parametrization given by Fuller, Fowler, and Newman [4] and subsequently used in the update of the presupernova electron capture rates by Aufderheide et al. [3]. We observe that Ref. [四] generally places the Gamow-Teller strength for even-even parent nuclei at too high an excitation energy in the daughter, while it is the opposite for odd- $A$ parent nuclei. The same trend as in the SMMC distributions is found in the data and has already been pointed out in Ref. [37. From the SMMC calculations and the data, we can conclude that for an even-even parent, the Gamow-Teller strength, at low temperatures, is at low daughter excitation energies $(\sim 2 \mathrm{MeV})$, while it is at significantly higher excitation energies $(\sim 5 \mathrm{MeV})$ for an odd- $A$ nucleus. This different behavior is related to the pairing energy difference between the even-even parent and odd-odd daughter. As the Gamow-Teller distributions usually have widths of order 1-2 MeV, our SMMC calculations for odd- $A$ nuclei might miss weak Gamow-Teller transitions at low excitation energies which could potentially increase the electron capture rates. It appears therefore reasonable to follow the prescription of 国 and add experimentally known transitions at low energies to the rates obtained from the SMMC Gamow-Teller distributions for odd- $A$ nuclei. Such a procedure seems to be unnecessary for even-even parent nuclei.

The electron capture cross sections calculated from the Gamow-Teller matrix elements are presented in Fig. 3 as a function of the incident electron energy $E_{e}$. The general behavior of the cross section can be easily understood. To trigger the electron capture process requires a minimum electron energy given by the mass splitting between parent and daughter, $Q_{i f}$. (This threshold is lowered by the internal excitation energy at finite temperature.) In eveneven parent nuclei the Gamow-Teller strength, at low temperatures, is centered at daughter excitation energies of order $2 \mathrm{MeV}$. Thus the electron capture cross sections for these parent nuclei increase drastically within the first couple of $\mathrm{MeV}$ of electron energies above threshold, reflecting the Gamow-Teller distribution. With increasing electron energies it continues to raise modestly caused by the $\left(E_{e}-\xi\right)^{2}$ factor in the cross section integral (12). As $Q_{i f}$ increases with neutron excess, caused by the increase of the symmetry energy, electron capture cross sections, at fixed electron energies, decrease with neutron excess. In odd$A$ nuclei the Gamow-Teller distribution, at low temperatures, peaks at noticeably higher daughter excitation energies. Thus the electron capture cross sections are shifted to higher electron energies for odd- $A$ nuclei in comparison to even-even parent nuclei by about $3 \mathrm{MeV}$, reflecting the difference in the Gamow-Teller peak positions.

Shown in Fig. 5 are the electron capture rates as a function of the electron chemical potential. We have used $T_{e}=T_{N}=0.5 \mathrm{MeV}$ for the electron capture rates on even parent 
nuclei, while for odd- $A$ parent nuclei we used $T_{N}=0.8 \mathrm{MeV}$ for the reasons discussed above. The electron chemical potential depends on the electron density, the electron fraction in the medium, and the temperature of the electrons. In the precollapse phase of supernova, a reasonable approximation is given by 38

$$
\mu_{e}=1.11\left(\rho_{7} Y_{e}\right)^{1 / 3}\left[1+\left(\frac{\pi}{1.11}\right)^{2} \frac{T_{e}^{2}}{\left(\rho_{7} Y_{e}\right)^{2 / 3}}\right]^{-1 / 3},
$$

where $T_{e}$ is in units of $\mathrm{MeV}$, and the electron density $\rho_{7}=\rho / 10^{7}$. Thus, for the nuclei studied here $\mu_{e} \approx 1.5-2 \mathrm{MeV}$ under typical presupernova conditions $\left(T_{N} \approx 0.4 \mathrm{MeV}, \rho_{7} \approx 6\right)$.

Do the present electron capture rates indicate potential implications for the pre-collapse evolution of a type II supernova? To make a judgement on this important question, we compare in Table II the SMMC rates for selected nuclei with those currently used in collapse calculations [3]. For the comparison we choose the same physical conditions as assumed in Tables 4-6 in [3]. Furthermore the table lists the partial electron capture rate which has been attributed to Gamow-Teller transitions in Ref. [3]. At first we note that for even parent nuclei, the present rate approximately agrees with the currently recommended total rate. A closer inspection, however, shows significant differences between the present rate and the one attributed to the Gamow-Teller transition in [3]. As discussed above, the origin of this discrepancy is due to the fact that Ref. [4] places the Gamow-Teller resonance for even-even nuclei systematically at too high an excitation energy. Of course, this shortcoming has been corrected for in Refs. 四, 泡 by adding an experimentally known low-lying strength on top of the one attributed to Gamow-Teller transitions. However, the overall good agreement between the SMMC results for even-even nuclei and the recommended rates indicates that our SMMC approach also accounts correctly for this low-lying strength. This has already been deduced from the good agreement between SMMC Gamow-Teller distributions and data including the low-energy regime. We conclude therefore that, for even-even nuclei, the SMMC approach is able to predict the total electron capture rate rather reliably, even if no experimental data are available. We note that our SMMC rate is somewhat larger than the recommended rate for ${ }^{56} \mathrm{Fe}$ and ${ }^{60} \mathrm{Ni}$. In both cases the experimental Gamow-Teller distribution is known and agrees well with the SMMC results [13]. While the proposed increase of the rate for ${ }^{60} \mathrm{Ni}$ is not expected to have noticeable influence on the pre-collapse evolution, the increased rate for ${ }^{56} \mathrm{Fe}$ makes this nucleus an important contributor in the change of $Y_{e}$ during the collapse (see Table 15 of [3]).

Turning now to electron capture on odd- $A$ nuclei. Here we observe that the SMMC electron capture rate, derived from the Gamow-Teller distributions, is significantly smaller than the recommended total rate. As already stressed above, this is simply due to the fact that for odd- $A$ nuclei the Gamow-Teller transition peaks at rather high excitation energies in the daughter nucleus. The electron capture rate on odd- $A$ nuclei is therefore carried by weak transitions at low excitation energies. Comparing our rates to those attributed to GamowTeller transitions in Refs. [4.3] reveals that the latter have been, in general, significantly overestimated which is simply caused by the fact that the position of the Gamow-Teller resonance is usually put at too low excitation energies in the daughter (see Table I). For example, Ref. [3] attributes $87 \%$ of the total capture rate on ${ }^{55} \mathrm{Co}$ to Gamow-Teller transitions (upper part of Table II), while our calculation predicts the contribution of the Gamow-Teller strength distribution to be negligible. Thus, we recommend that the capture rate on ${ }^{55} \mathrm{Co}$ 
is significantly smaller (only $13 \%$ of the rate given in Table 4 of Ref. [3]). Our SMMC calculation implies that the Gamow-Teller transitions should not contribute noticeably to the electron capture rates on odd- $A$ nuclei at the low temperatures studied in Tables 14-16 in [3]. Thus, the rates for odd- $A$ nuclei given in these tables should generally be replaced by the non-Gamow-Teller fraction.

\section{DISCUSSION}

In this paper we presented a detailed description of the electron-capture cross section and rates calculated from the Gamow-Teller distributions obtained using the SMMC method. We demonstrated the validity of our odd- $A$ sampling technique in ${ }^{45} \mathrm{Sc}$, and we found very good agreement between the SMMC and the traditional approach for solutions of the shell model. Furthermore, the SMMC approach reproduces the measured Gamow-Teller strength distributions very well. In accord with the data, we find an odd-even dependence of the Gamow-Teller peak position in the daughter nucleus: while it is generally at low daughter excitation energy for even-even parent nuclei $(\sim 2 \mathrm{MeV})$, it is at noticeable higher energies for odd- $A$ parents $(\sim 5 \mathrm{MeV})$. These systematics are not reproduced by the parametrization of the Gamow-Teller resonance as adopted for the derivation of the currently recommended rates [4. [3]. This parametrization places the Gamow-Teller resonance usually at too high excitation energies for even-even parent while the position is too low for odd- $A$ parents. However, this shortcoming has been mainly overcome in the recommended rates by adding experimentally known strength at low excitation energies to the Gamow-Teller strength.

The presupernova collapse occurs at finite temperature and our SMMC approach, for the first time, allows to take thermal effects consistently into account. With increasing temperature, the Gamow-Teller distribution is broadened and shifted to lower transfer energies. However, at the rather low temperatures at which electron capture occurs on nuclei in the iron mass region, the temperature dependence of the Gamow-Teller strength distribution is rather unimportant. For even-even nuclei the distribution does not change too much at $T_{N}<0.6 \mathrm{MeV}$ due to the large pairing gap. For odd- $A$ nuclei, the Gamow-Teller strength resides at such high excitation energies that the modest temperature dependence of the strength does not affect the total rate. Thus it also does not matter that numerical problems do not allow us to cool odd- $A$ nuclei below $T_{N}=0.8 \mathrm{MeV}$. However, we like to stress that the temperature dependence of the Gamow-Teller strength will become important at later stages of the presupernova collapse when temperatures of order $T_{N}=1 \mathrm{MeV}$ or higher are reached, as then the strength for both even-even and odd- $A$ nuclei is noticeably shifted downwards in transfer energy. At these temperatures the $Y_{e}$ value, however, has decreased enough so that electrons are captured on nuclei with $Z<40$ and $N>40$ for which the Gamow-Teller transitions are naively expected to be Pauli-blocked. However, this Pauli-blocking can be overcome by thermal excitation [39] and by pairing [40], which, at low temperatures, is the more important effect.

As the Gamow-Teller strength resides at rather high excitation energies for odd- $A$ parents, the Gamow-Teller contribution is generally negligible in the total electron capture rate. This finding is at variance with the recent compilations [4, 3] which propose noticeable Gamow-Teller fractions for the capture rate on odd- $A$ nuclei like ${ }^{55,59} \mathrm{Co}$. We recommend to use only the non-Gamow-Teller fraction of the compiled rates for odd- $A$ nuclei. For even- 
even parents, the Gamow-Teller distribution is located at very low excitation energies and, within our approach, should account for the total electron capture rate. In fact, our rate agrees reasonably well with the compiled total rates. However, for nuclei like ${ }^{56} \mathrm{Fe}$ and ${ }^{60} \mathrm{Ni}$, the Gamow-Teller strength resides, in agreement between data and SMMC calculation [13, at such low energies that the present rate is significantly larger than the one given in the compilations.

In summary, the present SMMC approach allows for the first time a microscopic determination of the Gamow-Teller contributions to the presupernova electron capture rates. The present calculation will be extended to other nuclei of potential importance during the collapse phase of type II supernova. The SMMC approach can also be extended to heavier, more neutron-rich nuclei which will carry the electron capture process at later stages. For these nuclei we expect the ability of our method to consistently account for finite temperature effects to be quite important.

\section{ACKNOWLEDGMENTS}

This work was supported in part through grant DE-FG02-96ER40963 from the U.S. Department of Energy. Oak Ridge National Laboratory (ORNL) is managed by Lockheed Martin Energy Research Corp. for the U.S. Department of Energy under contract number DE-AC05-96OR22464. KL has been partly supported by the Danish Research Council. Grants of computational resources were provided by the Center for Advanced Computational Research at Caltech, the Center of Computational Science at ORNL, and NERSC. 


\section{REFERENCES}

[1] F.K. Sataria and A. Ray, Phys. Rev. Lett. 79, 1599 (1997)

[2] H.A. Bethe, Rev. Mod. Phys. 62801 (1990)

[3] M. B. Aufderheide, I. Fushiki, S. E. Woosley, and D. H. Hartmann, Astrophys. J. Suppl. 91, 389 (1994)

[4] G.M. Fuller, W.A. Fowler and M.J. Newman, ApJS 42, 447 (1980); 48, 279 (1982); ApJ 252 (1982) 715; 293, 1 (1985)

[5] A.L. Williams et al., Phys. Rev. C51, 1144 (1995)

[6] W.P. Alford et al., Nucl. Phys. A514, 49 (1990)

[7] M.C. Vetterli et al., Phys. Rev. C40, 559 (1989)

[8] S. El-Kateb et al., Phys. Rev. C49, 3129 (1994)

[9] T. Rönnquist et al., Nucl. Phys. A563, 225 (1993)

[10] M.B. Aufderheide, Nucl. Phys. A526, 161 (1991)

[11] M.B. Aufderheide, S.D. Bloom, D.A. Ressler and G.J. Mathews, Phys. Rev. C47, 2961 (1993)

[12] M.B. Aufderheide, S.D. Bloom, D.A. Ressler and G.J. Mathews, Phys. Rev. C48, 1677 (1993)

[13] P.B. Radha, D.J. Dean, S.E. Koonin, K. Langanke, and P. Vogel, Phys. Rev. C56, 3079 (1997)

[14] C. W. Johnson, S. E. Koonin, G. H. Lang, and W. E. Ormand, Phys. Rev.. Lett. 69, 3157 (1992)

[15] G. H. Lang, C. W. Johnson, S. E. Koonin, and W. E. Ormand, Phys. Rev. C 48, 1518 (1993)

[16] W. E. Ormand, D. J. Dean, C. W. Johnson, G. H. Lang, and S. E. Koonin, Phys. Rev. C C49, 1422 (1994)

[17] S. E. Koonin, D. J. Dean, and K. Langanke, Phys. Rep. 278, 1 (1997)

[18] J. Hubbard, Phys. Rev. Lett. 3, 77 (1959)

[19] R. Stratonovich, Dokl. Akad. Nauk. SSSR 115, 1097 (1957)

[20] N. Metropolis, A. Rosenbluth, M. Rosenbluth, A. Teller, and E. Teller, J. Chem. Phys. 21, 1087 (1953)

[21] Y. Alhassid, D. J. Dean, S. E. Koonin, G. Lang, and W. E. Ormand, Phys. Rev. Lett. 72, 613 (1994)

[22] D. J. Dean, S. E. Koonin, K. Langanke, P. B. Radha, and Y. Alhassid, Phys. Rev. Lett 74, 2909 (1995)

[23] D.J. Dean, B.P. Radha, K. Langanke, S.E. Koonin, Y. Alhassid, and W.E. Ormand, Phys. Rev. Lett, 72, 4066 (1994)

[24] J.S. O'Connell, T.W. Donnelly, and J.D. Walecka, Phys. Rev. C6, 719 (1972)

[25] K. Langanke, D. J. Dean, P. B. Radha, Y. Alhassid, and S. E. Koonin, Phys. Rev. C 52, 718 (1995)

[26] E. Caurier, A. Zuker, A. Poves, and G. Martinez-Pinedo, Phys. Rev. C 50, 225 (1994)

[27] B. Brown and B. Wildenthal, Ann. Rev. Nucl. Part. Sci. 38, 29 (1988)

[28] G. Martinez-Pinedo, A. Poves, E. Caurier, and A. P. Zuker, Phys. Rev. C 53, R2602 (1996)

[29] G. Martinez-Pinedo, A.P. Zuker, A. Poves, and E. Caurier, Phys. Rev. C55, 187 (1997)

[30] A. Poves and A. Zuker, Phys. Rep. 70, 235 (1981) 
[31] T. T. S. Kuo and G. E. Brown, Nucl. Phys. A 114, 241 (1968)

[32] W.P. Alford et al, Nucl. Phys. A531, 97 (1991)

[33] G. Martinez-Pinedo, private communication

[34] T. Otsuka, M. Honma and T. Mizusaki, Phys. Rev. Lett., in print

[35] W. A. Richter, M. G. Vandermerwe, R. E. Julies, and B. A. Brown, Nucl. Phys. A523 (1991) 325

[36] D.J. Dean, B.P. Radha, K. Langanke, S.E. Koonin, Y. Alhassid, and W.E. Ormand, Phys. Rev. Lett, 72, 4066 (1994)

[37] S.E. Koonin and K. Langanke, Phys. Lett B326, 5 (1994)

[38] S.A. Bludman and K.A. van Riper, Astrophys. J. 224, 631 (1978)

[39] J. Cooperstein and J. Wambach, Nucl. Phys. A420, 591 (1984)

[40] K. Langanke, E. Kolbe and D.J. Dean, in preparation 


\section{TABLES}

TABLE I. Comparisons of the positions of the Gamow-Teller peaks as calculated within the SMMC approach with the parametrization of Ref. 44. The proton and neutron numbers refer to the parent nucleus, while $E_{G T}$ is the position of the Gamow-Teller peak in the daughter nucleus. (To obtain this number we have shifted the transferred energy $E$ by the experimental mass splitting between parent and daughter nucleus.) $E_{\mathrm{FFN}}$ denotes the position of the Gamow-Teller resonance due to the parametrization given in Ref. [4] and subsequently used in presupernova collapse studies. The SMMC calculations have been performed at $T_{N}=0.5 \mathrm{MeV}$ for even nuclei and at $T_{N}=0.8$ $\mathrm{MeV}$ for odd- $A$ nuclei. For ${ }^{50} \mathrm{Cr}$ the single-particle model allows transitions into the $f_{7 / 2}$ and $f_{5 / 2}$ orbitals.

\begin{tabular}{|c|c|c|c|}
\hline \hline $\mathrm{N}$ & $\mathrm{Z}$ & $E_{G T}$ & $E_{\mathrm{FFN}}$ \\
\hline 28 & 28 & 2.6 & 3.78 \\
30 & 28 & 2.0 & 3.76 \\
32 & 28 & 1.0 & 2.0 \\
28 & 27 & 6.9 & 3.78 \\
30 & 27 & 4.8 & 3.77 \\
32 & 27 & 4.2 & 2.0 \\
28 & 26 & 2.8 & 3.80 \\
29 & 26 & 6.1 & 5.4 \\
30 & 26 & 1.5 & 3.78 \\
32 & 26 & -0.5 & 2.0 \\
30 & 25 & 4.7 & 3.79 \\
26 & 24 & 2.5 & $2.0,8.7$ \\
28 & 24 & 1.3 & 3.82 \\
28 & 23 & 5.5 & 3.83 \\
\hline \hline
\end{tabular}


TABLE II. Comparisons of the present SMMC electron capture rates with the total $\left(\lambda_{\mathrm{ec}}\right)$ and partial Gamow-Teller $\left(\lambda_{\mathrm{ec}}^{\mathrm{GT}}\right)$ rates as given in Ref. [3]. Physical conditions at which the comparisons were made are given in the last column.

\begin{tabular}{|c|c|c|c|c|}
\hline nucleus & $\begin{array}{c}\lambda_{\mathrm{ec}}\left(\mathrm{sec}^{-1}\right) \\
(\mathrm{SMMC})\end{array}$ & $\begin{array}{c}\lambda_{\mathrm{ec}}\left(\mathrm{sec}^{-1}\right) \\
(\text { Ref. } \mid 3 \|)\end{array}$ & $\begin{array}{c}\lambda_{\mathrm{ec}}^{G T}\left(\mathrm{sec}^{-1}\right) \\
\text { (Ref. } \mid 3 \|)\end{array}$ & conditions \\
\hline${ }^{55} \mathrm{Co}$ & $2.25 \mathrm{E}-04$ & $1.41 \mathrm{E}-01$ & $1.23 \mathrm{E}-01$ & $\rho_{7}=5.86, T_{9}=3.40, Y_{e}=0.47$ \\
\hline${ }^{57} \mathrm{Co}$ & $1.97 \mathrm{E}-06$ & $3.50 \mathrm{E}-03$ & $1.31 \mathrm{E}-04$ & $\rho_{7}=5.86, T_{9}=3.40, Y_{e}=0.47$ \\
\hline${ }^{54} \mathrm{Fe}$ & $4.64 \mathrm{E}-05$ & $3.11 \mathrm{E}-04$ & $9.54 \mathrm{E}-07$ & $\rho_{7}=5.86, T_{9}=3.40, Y_{e}=0.47$ \\
\hline${ }^{55} \mathrm{Fe}$ & 7.22E-09 & $1.61 \mathrm{E}-03$ & $1.16 \mathrm{E}-07$ & $\rho_{7}=5.86, T_{9}=3.40, Y_{e}=0.47$ \\
\hline${ }^{56} \mathrm{Ni}$ & $1.96 \mathrm{E}-02$ & $1.60 \mathrm{E}-02$ & $6.34 \mathrm{E}-03$ & $\rho_{7}=5.86, T_{9}=3.40, Y_{e}=0.47$ \\
\hline${ }^{58} \mathrm{Ni}$ & $6.02 \mathrm{E}-04$ & $6.36 \mathrm{E}-04$ & $4.04 \mathrm{E}-06$ & $\rho_{7}=5.86, T_{9}=3.40, Y_{e}=0.47$ \\
\hline${ }^{60} \mathrm{Ni}$ & $1.34 \mathrm{E}-05$ & $1.49 \mathrm{E}-06$ & $4.86 \mathrm{E}-07$ & $\rho_{7}=5.86, T_{9}=3.40, Y_{e}=0.47$ \\
\hline${ }^{59} \mathrm{Co}$ & $2.05 \mathrm{E}-07$ & 2.09E-04 & $6.37 \mathrm{E}-05$ & $\rho_{7}=10.7, T_{9}=3.65, Y_{e}=0.455$ \\
\hline${ }^{57} \mathrm{Co}$ & $1.21 \mathrm{E}-05$ & $7.65 \mathrm{E}-03$ & $3.69 \mathrm{E}-04$ & $\rho_{7}=10 . .7, T_{9}=3.65, Y_{e}=0.455$ \\
\hline${ }^{55} \mathrm{Fe}$ & $6.48 \mathrm{E}-08$ & $3.80 \mathrm{E}-03$ & $5.51 \mathrm{E}-07$ & $\rho_{7}=10.7, T_{9}=3.65, Y_{e}=0.455$ \\
\hline${ }^{56} \mathrm{Fe}$ & $5.86 \mathrm{E}-06$ & $4.68 \mathrm{E}-07$ & $6.60 \mathrm{E}-10$ & $\rho_{7}=10.7, T_{9}=3.65, Y_{e}=0.455$ \\
\hline${ }^{54} \mathrm{Fe}$ & $2.26 \mathrm{E}-04$ & $9.50 \mathrm{E}-04$ & $3.85 \mathrm{E}-06$ & $\rho_{7}=10.7, T_{9}=3.65, Y_{e}=0.455$ \\
\hline${ }^{51} \mathrm{~V}$ & $6.17 \mathrm{E}-07$ & $1.24 \mathrm{E}-05$ & $9.46 \mathrm{E}-09$ & $\rho_{7}=10.7, T_{9}=3.65, Y_{e}=0.455$ \\
\hline${ }^{52} \mathrm{Cr}$ & $5.22 \mathrm{E}-07$ & $2.01 \mathrm{E}-07$ & $1.59 \mathrm{E}-10$ & $\rho_{7}=10.7, T_{9}=3.65, Y_{e}=0.455$ \\
\hline${ }^{60} \mathrm{Ni}$ & $7.48 \mathrm{E}-05$ & $7.64 \mathrm{E}-06$ & $2.12 \mathrm{E}-06$ & $\rho_{7}=10.7, T_{9}=3.65, Y_{e}=0.455$ \\
\hline
\end{tabular}




\section{FIGURES}

FIG. 1. Top panel: The Gamow-Teller transitions as a function of the energy transfer $E=E_{f}-E_{i}$ using SMMC for ${ }^{45} \mathrm{Sc}$, and comparing to diagonalization calculations (DD) at $T_{N}=0.33$ and $0.8 \mathrm{MeV}$. Middle panel: The cross section calculated from both SMMC and diagonalization. Bottom panel: Calculated rates from SMMC $\left(T_{N}=0.8 \mathrm{MeV}\right)$, assuming $T_{e}=0.3$ and $0.8 \mathrm{MeV}$, compared to the exact results $\left(T_{e}=T_{N}\right)$.

FIG. 2. The error band in the rates calculations for ${ }^{45} \mathrm{Sc}$, where the position of the calculated Gamow-Teller centroid has been shifted by $0.5 \mathrm{MeV}$.

FIG. 3. Shown are the $G T_{+}$distributions for various nuclei studied here as a function of the

energy transfer from the electron, $E=E_{f}-E_{i}$. These curves were obtained after MaxEnt reconstruction of the Gamow-Teller response functions that were calculated in the SMMC framework.

FIG. 4. Shown are the electron capture cross sections as a function of the incident electron energy $E_{e}$ for all nuclei in this study.

FIG. 5. Shown are the electron capture rates as a function of the electron chemical potential energy $\mu_{e}$ for all nuclei in this study. All electron temperatures were fixed at $T_{e}=0.5 \mathrm{MeV}$. 

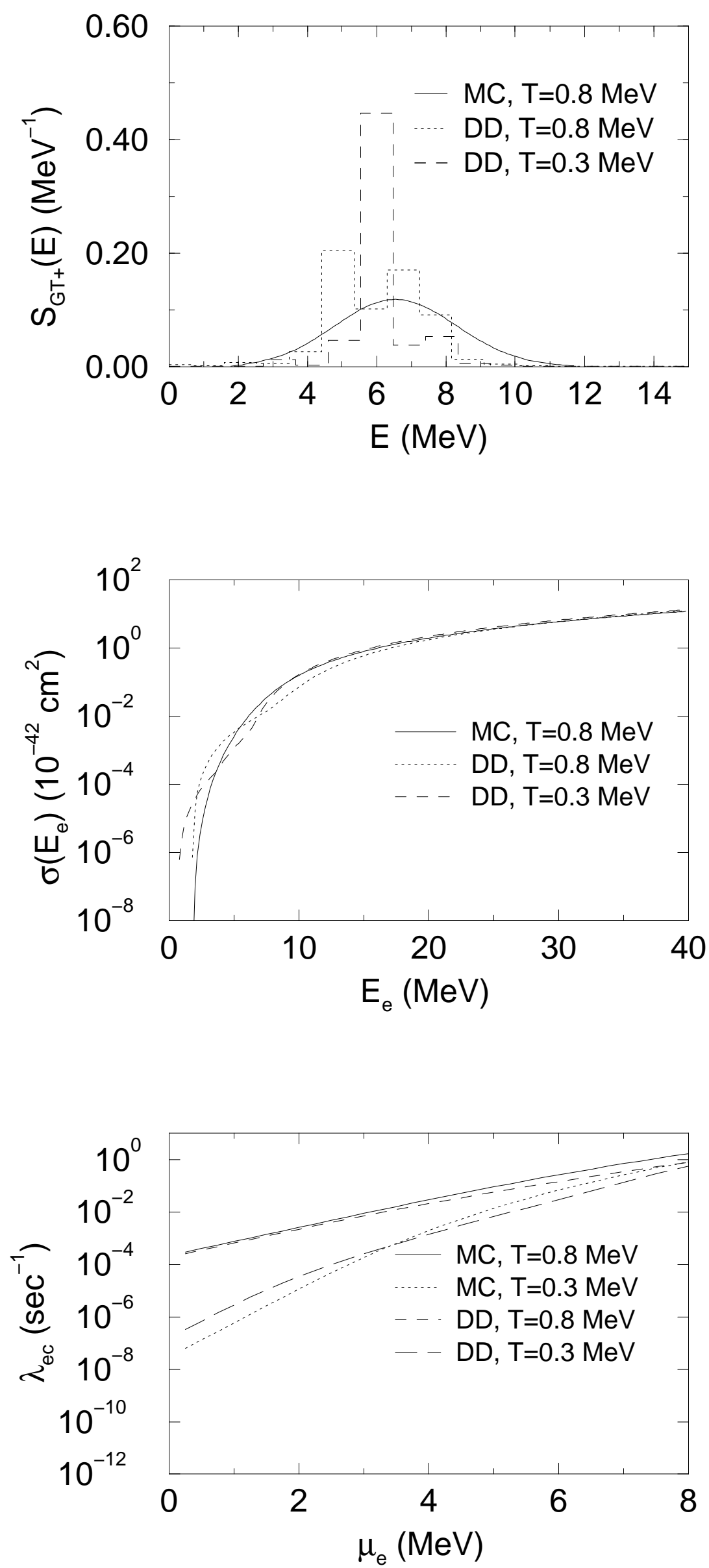


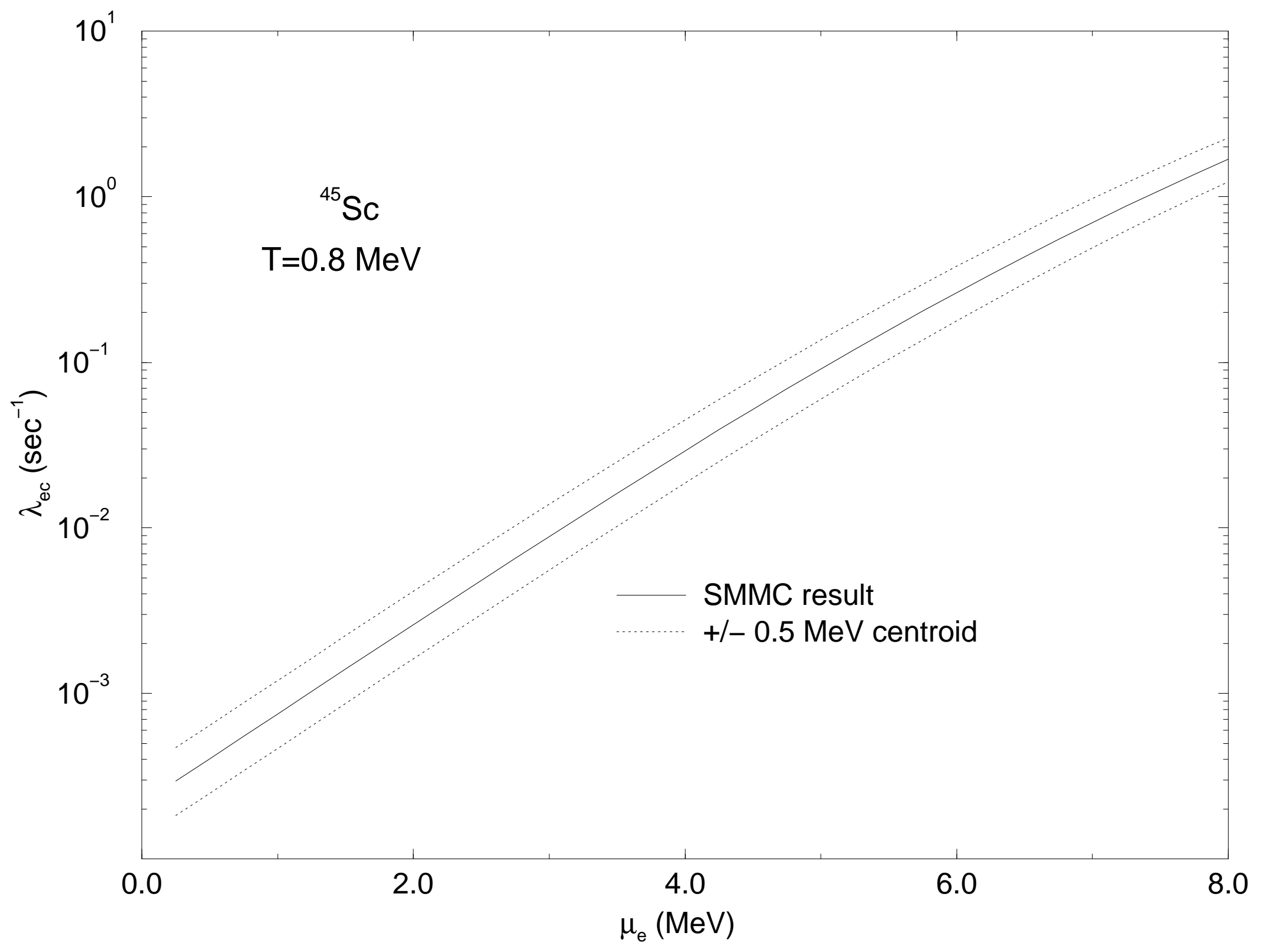



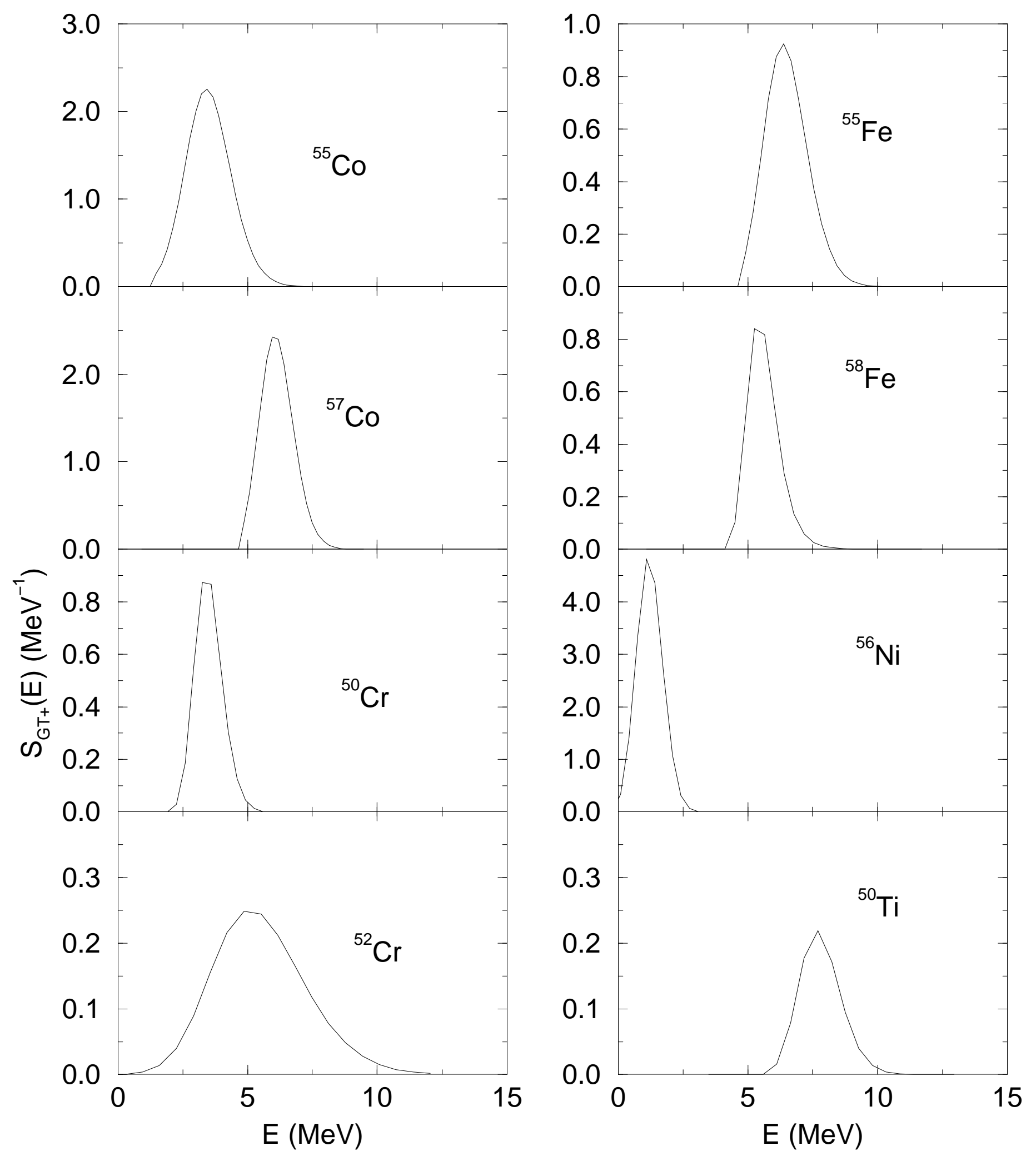

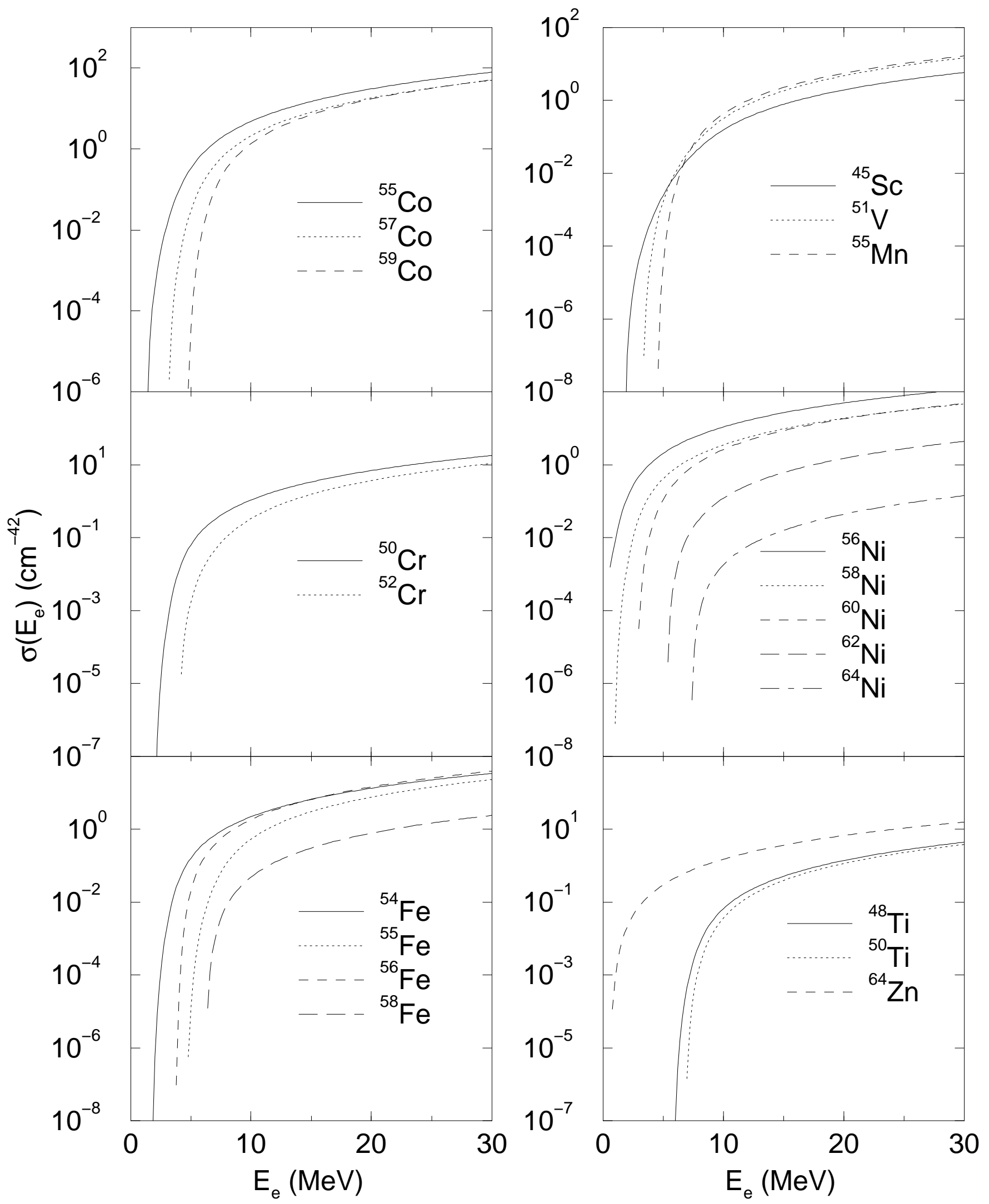

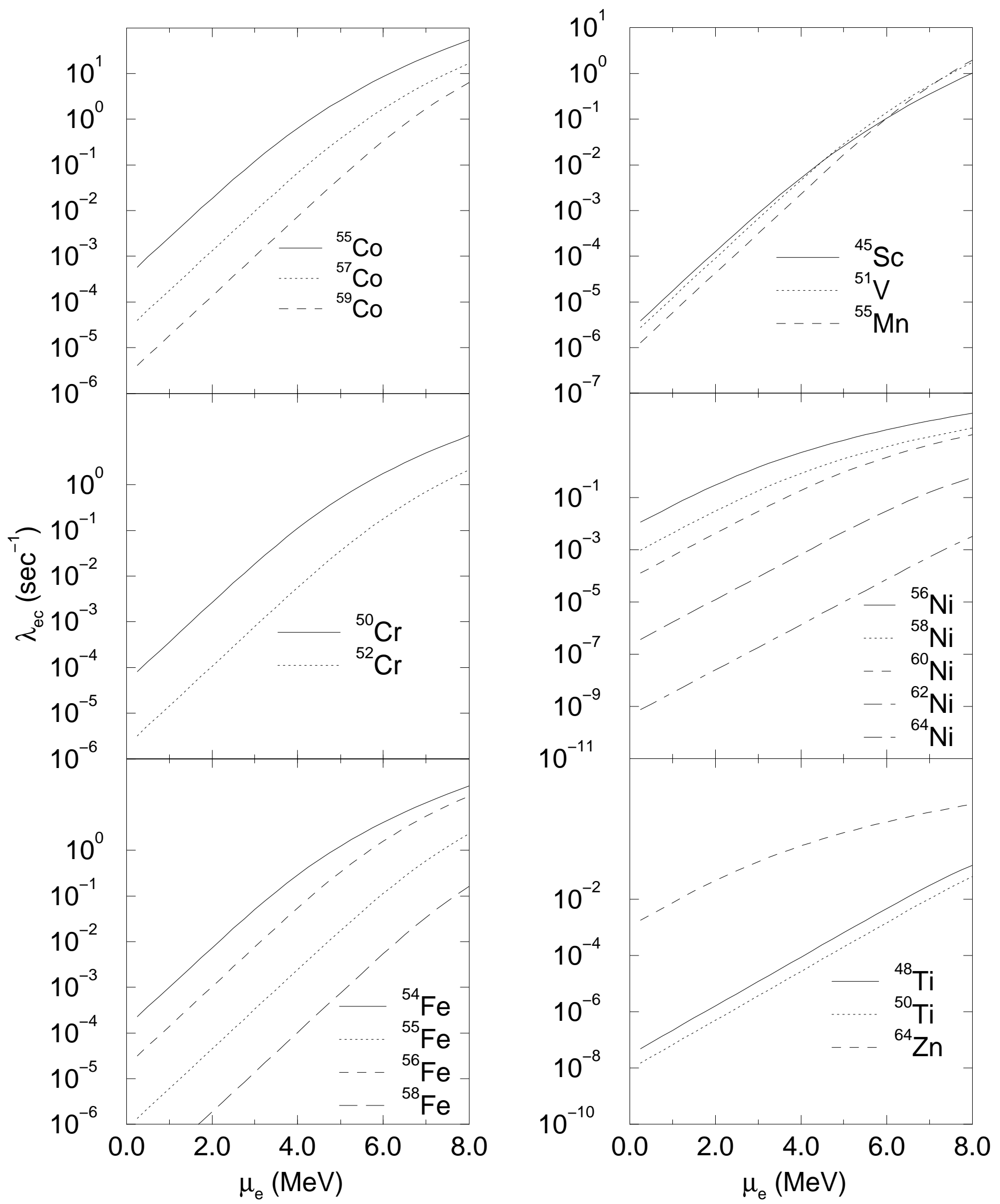